\title{
Efficient execution of ATL model transformations using static analysis and parallelism
}

\author{
Jesús Sánchez Cuadrado, Loli Burgueño, Manuel Wimmer, and Antonio Vallecillo
}

\begin{abstract}
Although model transformations are considered to be the heart and soul of Model Driven Engineering (MDE), there are still several challenges that need to be addressed to unleash their full potential in industrial settings. Among other shortcomings, their performance and scalability remain unsatisfactory for dealing with large models, making their wide adoption difficult in practice. This paper presents $\mathrm{A} 2 \mathrm{~L}$, a compiler for the parallel execution of ATL model transformations, which produces efficient code that can use existing multicore computer architectures, and applies effective optimizations at the transformation level using static analysis. We have evaluated its performance in both sequential and multi-threaded modes obtaining significant speedups with respect to current ATL implementations. In particular, we obtain speedups between $2.32 x$ and $38.28 x$ for the $A 2 L$ sequential version, and between $2.40 x$ and $245.83 x$ when $A 2 L$ is executed in parallel, with expected average speedups of $8.59 x$ and $22.42 x$, respectively.
\end{abstract}

Index Terms-Model transformation, MDE, ATL, Performance, Scalability, Parallelization

\section{Introduction}

The progressive adoption of Model-Driven Engineering (MDE) 1] approaches for developing better and more efficient software is posing different kinds of challenges to current MDE methods and tools. Despite the potential benefits of MDE technologies to significantly reduce time to market and improve product quality, they still suffer from some limitations that may hinder their full adoption by industry (see, e.g., 2][4]). In particular, the scalability, usability and performance of model transformations (MT) are crucial issues that need to be tackled if they are to be effectively used to address scenarios such as model-driven modernization of legacy systems and the engineering of large and complex applications in, e.g., the automotive, biology or aerospace domains.

At this moment, ATL [5] and QVT 6] are the most widelyused model transformation languages 7. Although they provide powerful abstractions to specify and implement transformations between models and to generate model views, their implementations have limited scalability, and thus the execution time of transformations may become prohibitive with large input models (e.g., in the order of millions of elements), or even medium-size input models if the transformation has complex model navigations. One reason for this lack of scalability is due to the fact that most transformation engines are implemented as simple interpreters and they barely use static

- Jesús Sánchez Cuadrado is with the Universidad de Murcia, Dept. Informática y Sistemas. Campus de Espinardo, Murcia, Spain E-mail: jesusc@um.es

- Loli Burgueño is with the Open University of Catalonia, IN3, Barcelona, Spain, and Institut LIST, CEA, Université ParisSaclay, París, France

Email: lburguenoc@uoc.edu

- Antonio Vallecillo is with ITIS Software, Universidad de Málaga, Bulevar Louis Pasteur, 35, 29071, Malaga, Spain

E-mail:av@lcc.uma.es

- Manuel Wimmer is with the Johannes Kepler Universität, Business Informatics - Software Engineering, Linz, Austria

E-mail:manuel.wimmer@jku.at analysis information to apply compile time optimizations or to improve their scheduling. Moreover, although multicore computers are widely available, there are very few engines that implement parallel transformation algorithms.

The contribution presented in this paper addresses the engineering of an efficient model transformation engine for the particular case of the ATL model transformation language. We have developed a new compiler for ATL, called A2L, which provides several novel features with respect to state-of-the-art approaches, namely:

- A2L uses static analysis information provided by AnATLyzer [8] to compile ATL transformations to the Java Virtual Machine (JVM), applying optimizations for OCL expressions and for transformation rule handling.

- We present a novel algorithm which enables the parallel execution of the transformation, using data parallelism. This allows A2L to achieve an effective distribution of the parallel jobs, thus outperforming other parallel ATL engines which are based on task parallelism [9], 10].

- A2L is integrated with the ATL/AnATLyzer IDE and Eclipse Java Development Toolkit (JDT), which enables the development of transformations using the facilities provided by ANATLYZER, e.g., quick fixes 11] and visualizations 12. Moreover, the compiled code can be seamlessly integrated with existing Java code.

A2L has been validated for correctness using the regression tests defined for the ATL virtual machine 13 and supports the majority of the constructs of ATL, including all types of rules (matched, lazy and called), module and context helpers, imperative blocks, all datatypes including collections, maps and tuples, and the standard OCL library. We have run several benchmarks that show significant performance improvements when compared with the existing ATL engines.

This paper is organized as follows. Sect. 2 introduces the ATL model transformation language and describes the limi- 
tations of current transformation engines through a running example. Then, Sect. 3 describes the architecture of A2L, the compiler we have developed to compile and execute in parallel existing ATL programs. The more prominent features of A2L are described in Sections 4 and 5 , which present, respectively, the algorithm used to execute ATL transformations in parallel, and the A2L optimization strategies and mechanisms enabled by the use of ANATLYZER static typing information. Sect. 6 describes the evaluation that we have conducted to validate our proposal. Finally, Sect. 7 discusses related work, and Sect. 8 concludes with an outline on future work.

\section{Motivation and background}

Performance and scalability of model transformations is deemed as one of the most important challenges in MDE since it enables the use of model transformation technology to handle large models appearing in scenarios like reverse engineering, model analysis, or data engineering. It is also key to apply MDE to other engineering disciplines, such as construction [14] or automotive engineering [15].

Model transformation languages, notably those with a declarative form, have the potential to tackle this challenge because they provide an abstraction to write transformations which are independent of the execution mechanism. A good compiler should generate efficient code by analysing the structure and relationships of the transformation. However, this possibility has not been exploited in state-of-the-art MT languages, resulting in poor performance. In fact, a recent study [7] has revealed that, although MT users value the advantages of using MT languages, the poor performance and scalability issues of MT engines are hampering their use and forcing them to develop their transformations in generalpurpose languages (even though if it makes the task more cumbersome and error-prone).

Our working hypothesis is twofold. First, by using static analysis information, it is possible to compile declarative transformations to produce high-performance code; moreover, recurrent transformation idioms can be optimized by the compiler. Second, since a declarative transformation does not prescribe the execution order, it is possible to seamlessly execute a transformation in parallel, if the adequate transformation algorithm is implemented. More precisely, to achieve efficient parallel execution of ATL programs, such an algorithm should limit the number of dependencies between parallel processes to maximize concurrency, while load balancing between processes should aim at preventing processes from becoming idle if they finish before others. To this end, we propose the use of data-based parallelism, whereby the model is split into chunks of elements that are transformed by the processes, all running the complete transformation in parallel.

This paper describes our proposed parallelization algorithm, its main features and characteristics, the optimizations we have applied, and the performance gains it achieves over existing ATL model transformation engines.

\section{$2.1 \quad$ ATL}

ATL 5 is a hybrid model transformation language that allows both declarative and imperative constructs. A transformation consists of a set of rules that specifies which elements of the output model are created from which ones of the input model.

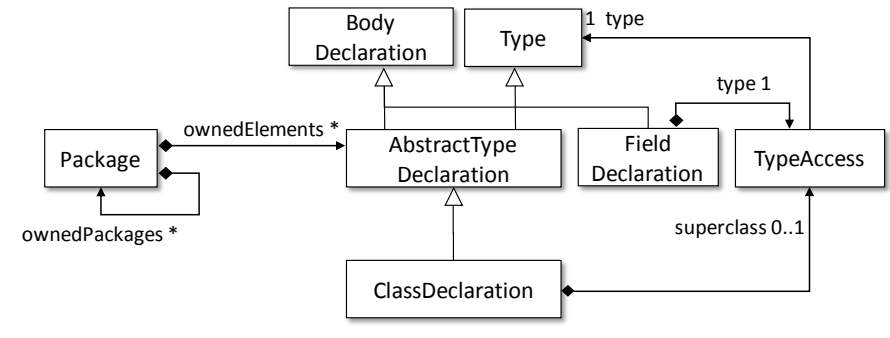

Fig. 1. Excerpt of the Java metamodel (MoDisco).

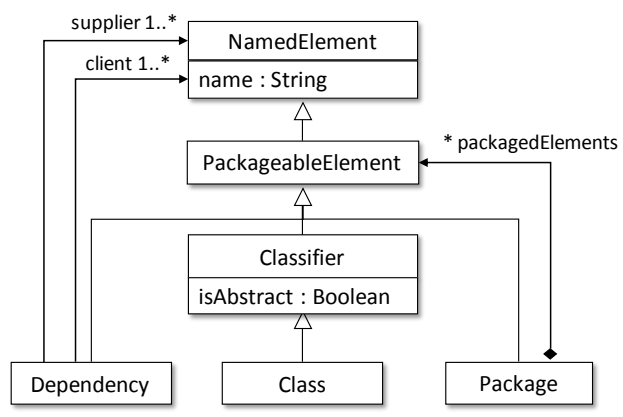

Fig. 2. Excerpt of the UML metamodel.

Listing 1 shows an excerpt of an ATL transformation taken from the ARTIST project [16] that generates a UML class diagram (a dependency view) from a Java project. Excerpts from the input and output meta-models of this transformation are depicted in Fig. 1 and 2 respectively. In ATL, the main type of rule is the so-called matched rule. It consists of an input pattern that might have a filter condition which is matched on the source model, and an output pattern that produces a set of elements in the target model for each matched input pattern. OCL expressions 17 are used to calculate the values of features of the target elements. In this excerpt, we have included two matched rules, Package2Package and Class2Class, which take package and class elements respectively from the Java model and convert them to the corresponding counterparts in the UML model, but filtering proxies out (not s1.proxy). A rule body consists of binding elements. A binding either assigns a primitive value (e.g., name $\leftarrow$ s1.name) or resolves the source values appearing in its right-hand side (RHS) to target values generated by other rules. For example, the binding in line 15 retrieves and assigns the subpackages mapped by rule Package2Package, and the binding in line 16 retrieves and assigns all non-proxy classes mapped by rule Class2Class.

The ATL transformation algorithm works in two phases, which are graphically illustrated in Fig. 3 The left-hand side shows a sample input model. In the first phase each matched rule accesses the input model to get all elements whose type is compatible with its input element (specified in the from part of the rules). The filter is used to rule elements out. In the example, elements jp1 and jp3 are retrieved by the rule Package2Package but only jp1 satisfies the filter and is matched. When an element is matched, the target elements specified in the to part of the rules are created and a traceability link is established (depicted by a dashed arrow in the image) which includes a reference to the rule producing the link. The second phase of the algorithm consists of traversing all traceability links and resolving each rule binding in order. To resolve a binding, its OCL expression in the RHS 
Listing 1. Excerpt of the Java2UML transformation.

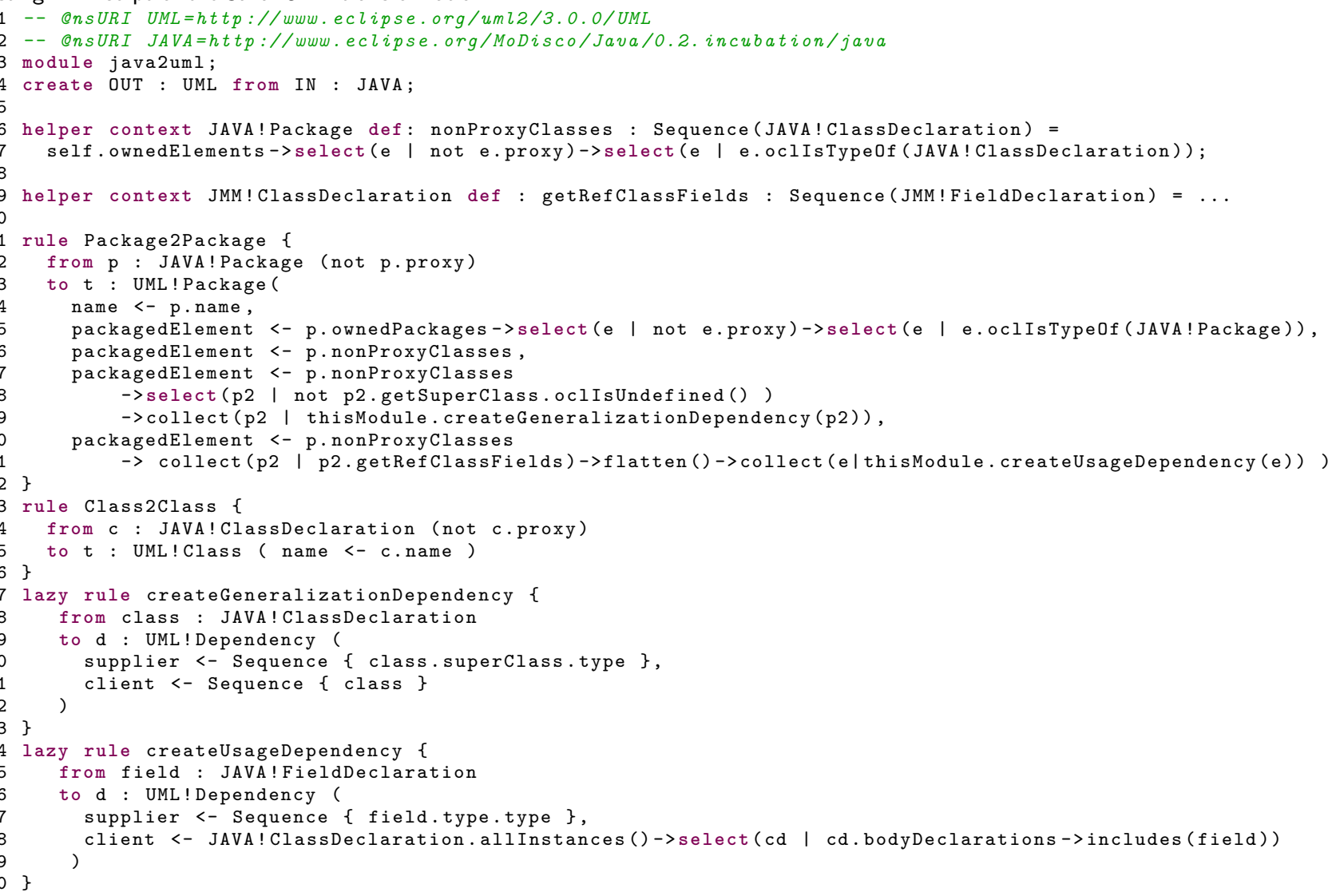

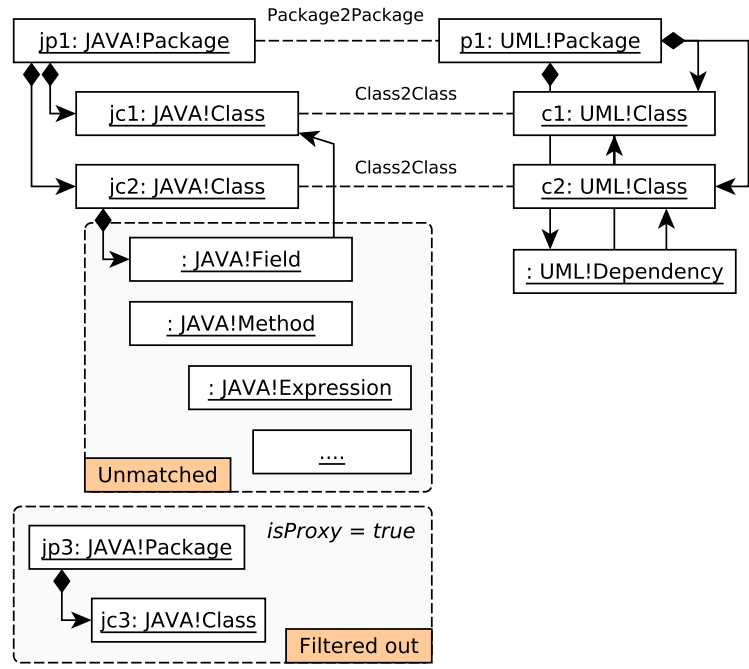

Fig. 3. Representation of a sample transformation execution.

is evaluated. If the result is a primitive type, the value is directly assigned to the feature in the left hand side. If it is an object (or a collection of objects), the internal trace is looked up to retrieve the corresponding target element and it is assigned to the left hand side (if it is a collection, the value is added). In the example, to resolve the binding in line 16 , the engine retrieves and assigns target objects $c 1$ and $c 2$ from source objects jc1 and jc2 respectively.
ATL also supports rules which must be explicitly invoked. This is the case of lazy rules. A lazy rule can be seen as a global function that takes model elements as parameters and returns a target model element, which is created and initialized by the rule. In the example, the lazy rule createUsageDependency (line 38) defines a dependency between the class that defines a field and the field type. In line 21, the rule is invoked. Since the lazy rule generates the target element, it can be assigned directly in the corresponding binding (i.e., no binding resolution is needed).

The ATL code is compiled into bytecode for its execution using two main runtime engines: the default ATL virtual machine [5], which was released along with the ATL language; and EMFTVM 18, which provides performance improvements as well as other advanced language features such as the possibility to execute in-place model transformations.

\subsection{Static analysis of model transformations: AnATLyzer}

A model transformation is typed against its input and output meta-models. This means that the types and features used in the transformation program must exist in the corresponding meta-model. This can be enforced dynamically (at runtime) or statically (at compilation time). Some languages, like QVTOperational, enforce this statically, while others, like ATL and Epsilon ETL 19, do it dynamically. In addition, data dependencies between the input model and the rules which match the elements, as well as among the transformation rules (i.e., established by means of bindings in the case of ATL), may exist. In this work, we have used AnATLYzer 8, 12 to 
statically analyse ATL transformations and benefit from this information to implement our compiler.

Fig. 4 illustrates some of the static analysis information made available by ANATLyzer. First, every node of the abstract syntax tree of the transformation is annotated with its type (i.e., a reference to the corresponding meta-model element). There are three bindings to initialize packagedElement, whose semantics is to add elements (i.e., the second binding does not override the previous setting because it is a collection). For example, the type of the first binding is Sequence (JAVA! Package) because AnATLYZER recognizes the use of oclIs TypeOf and performs an implicit casting. From the inferred types, AnATLyzer builds a graph to make dependencies among rules explicit. For instance, the first binding packagedElement can only be resolved by rule Package2Package because of the implicit casting that determines that the RHS of the binding will only have JAVA! Package elements. The second binding is resolved by the rule Class2Class because the return type of nonProxyClasses is Sequence (JAVA! ClassDeclaration). Finally, the third binding does not need to be resolved because it directly assigns target elements generated by the createUsageDependency rule.

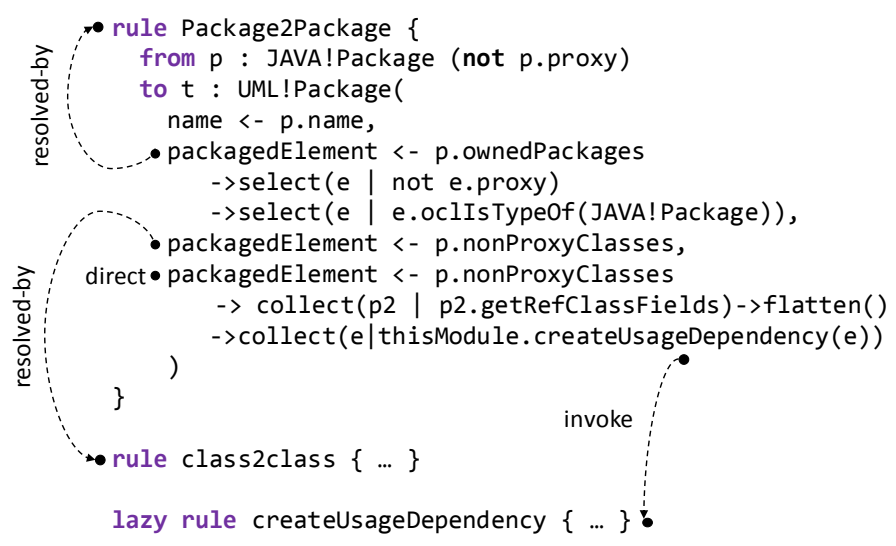

Fig. 4. Static analysis of the example transformation.

\subsection{Limitations of current approaches}

The original ATL transformation algorithm, based on the two phases described above, and its implementations (both in the standard ATL Virtual Machine (VM) [5] and EMFTVM [18]), can cope with scenarios involving small or medium-size models. However, their performance and scalability rapidly degrade as the size of the input models grows. Among other reasons, they fail to exploit a variety of interesting performance and optimization opportunities, which are described next.

Limited parallelism. The algorithm and its current implementations are sequential. A relatively simple approach to make the algorithm parallel is to use task parallelism [10, in which the parallelisation unit is the transformation rule. However, this approach is sub-optimal since it suffers from lock contention and unbalanced loads (i.e., some threads will be idle if they finish their tasks earlier than others). Using a data parallelism approach, all processes perform the same task, but on different chunks of data - it is the data that is split. Contrarily, in task parallelism, it is the model transformation that is split into separate smaller processes (e.g., a

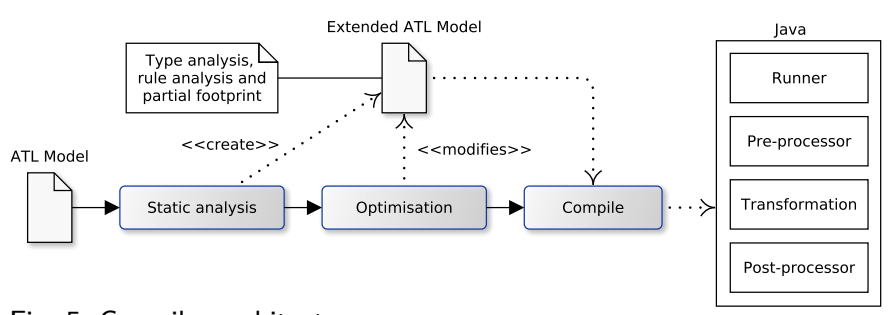

Fig. 5. Compiler architecture.

rule) and all of them work on the same data. As demonstrated in 20, using data parallelism to implement concurrent model transformations can produce significantly better results.

Inefficient model access. A transformation engine which does not exploit type information (such as the existing ATL virtual machines) does a "blind access" to the input model. This means that after loading the input, it cannot discard unused parts of the model. For instance, in the example (see Fig. 3), objects of types JAVA!Field and JAVA!Method will never be matched by a rule, and thus, they could be ruled out in the loading phase. We will improve rule matching by considering, in a pre-processing step, only those elements that are relevant for the transformation.

Expensive runtime checks. The ATL compiler does not perform any type checking, which means that it needs to insert code to perform dynamic checks, including, e.g., the cardinality of the LHS of the binding, or calls to helper methods. Moreover, it can only use the reflective EMF API, which also imposes an additional overhead. We will use the information made available by the type checker to avoid these kinds of overheads.

Lack of OCL optimizations. Complex transformations typically contain many OCL expressions and operation helpers. These expressions are often devoted to navigating collections. A good implementation of OCL is critical to achieve a satisfactory performance on large models - especially when collection operations are involved. It has already been reported that the standard ATL VM does not handle large collections efficiently 21 and it is the EMFTVM engine which does provide a better implementation. However, both engines have not addressed optimizations yet. For instance, the expression

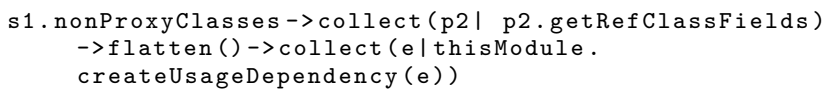

requires two intermediate collections to be created (for the first collect and the flatten). An optimizer could identify this pattern and evaluate the expression without creating unnecessary intermediate collections.

To the best of our knowledge, there is no transformation engine that makes use of static analysis information to improve its performance, and combines this with parallelism to take advantage of all the computing power of current CPUs.

\section{A2L: A Compiler for ATL}

Our technical approach to address the limitations presented above is based on a compiler from ATL to Java. Fig. 5 shows its architecture.

First, it performs static analyses using ANATLYzer. This produces an extended ATL abstract syntax model 
(AST), which includes type information, control flow and data flow information. This will be used throughout the compilation process.

The optimization phase (described in detail later in Sect. 5D is in charge of detecting common transformation patterns that can be particularly managed to generate efficient code. To implement the optimizations, special nodes are added to the AST. Each of these nodes represents an optimization pattern. The optimizer is in charge of detecting the patterns and replacing the AST nodes. Then, the compiler has specific extensions to produce specific code for these nodes. This optimization phase is optional and can be disabled.

The compilation phase generates all the code and related artefacts needed to execute the transformation in the JVM. An essential feature of A2L is that it targets a transformation algorithm specifically designed to execute the model transformations concurrently, using data parallelism to achieve adequate performance results (the algorithm is described in detail later in Sect. 4, and the gains in performance are presented in Sect. 6).

As a result of these three steps, the compiler generates four main artefacts (Fig. 5). The Runner allows the user to configure the transformation execution programatically (e.g., to set the input models, to configure the number of threads, etc.). The Pre-processor is in charge of filtering the input models to optimize the rule matching by considering only those elements required by the transformation rules. The Transformation contains the actual transformation behavior which will be executed by the parallel processes. Finally, the Post-processor is in charge of combining the results of all the processes that have been working in parallel to realize the transformation, and to generate the output models.

The following sections describe in more detail these features of the A2L compiler. We begin explaining our parallel transformation algorithm. Then, we explain the optimization strategies and mechanisms to implement them.

\section{Parallel execution of ATL transformations}

To address the lack of parallelism of ATL we have designed a new transformation algorithm. The algorithm is intended to respect the semantics of the original one but, in addition, it enables data-based parallelism and focuses on minimizing the amount of lock contention among the worker threads.

In data-based parallelism, all threads execute the same code but on different chunks of data. The advantage over task-based parallelism, as proposed in 10 for ATL, is that processors are less prone to be idle. We have redesigned the ATL transformation algorithm to make it amenable to databased parallelism, generalizing the approach proposed in 20 . Our algorithm is presented in Algorithm 1. It works in three phases, pre-processing (line 2), execution (line 5) and postprocessing (line 11). The architecture to execute these phases in parallel is illustrated in Fig. 6 and it is described next.

Pre-processing. The input model is read from some source (label 1). Its elements are placed in a buffer which will be used by worker threads in the next phase when fetching work. However, not all model elements are required by all worker threads, only those whose type is declared by the matched rules in their source patterns. Thus, this set of types is extracted from the static analysis of the transformation, and used to filter

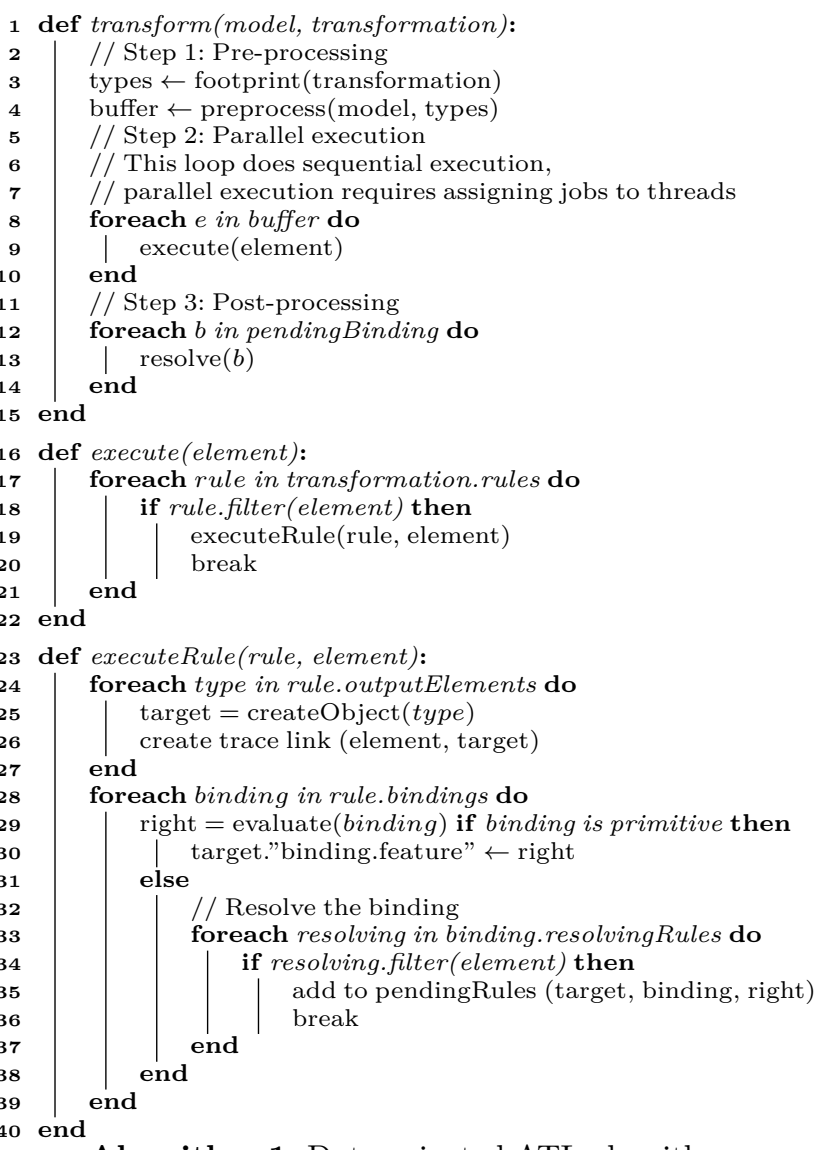

Algorithm 1: Data-oriented ATL algorithm.

the source model (lines 344). In the transformation example, this set consists of Package and ClassDeclaration types. The intended effect is to reduce the size of the buffer and to speed up rule matching, since there are less elements to consider. Although this step is done sequentially, the actual overhead due to the filtering is small since the engine needs to load and prepare the input model in any case.

Execution. This phase is in charge of executing the transformation logic. In the sequential version each element in the buffer is processed one after another. In the parallel version, we spawn worker threads (label 2). A worker obtains a chunk of data from the buffer (label 4), which is split into chunks of a given size (e.g., 512 model elements). Each worker has a counter to represent the chunk that is currently transforming. When it finishes, it asks for the next chunk to the scheduler, which uses an atomic integer variable to represent the last chunk given to a worker. The scheduler uses an atomic operation to increment the counter (label 3), which means that the increment operation for the chunk counter does not need to be guarded by a lock because it is done atomically. This way, there are no locks involved in the algorithm and we expect less contention. There are several possible strategies to split to decide the chunk size. The simplest one is to use a fixed chunk size. The larger the chunk size the less competition to get more work. However, it may happen that some threads are idle at the end of the transformation execution (i.e., load imbalance). On the contrary, setting a small chunk size would lead to more contention. The alternative is a dynamic scheduling policy in which chunks are larger at the beginning and smaller towards 


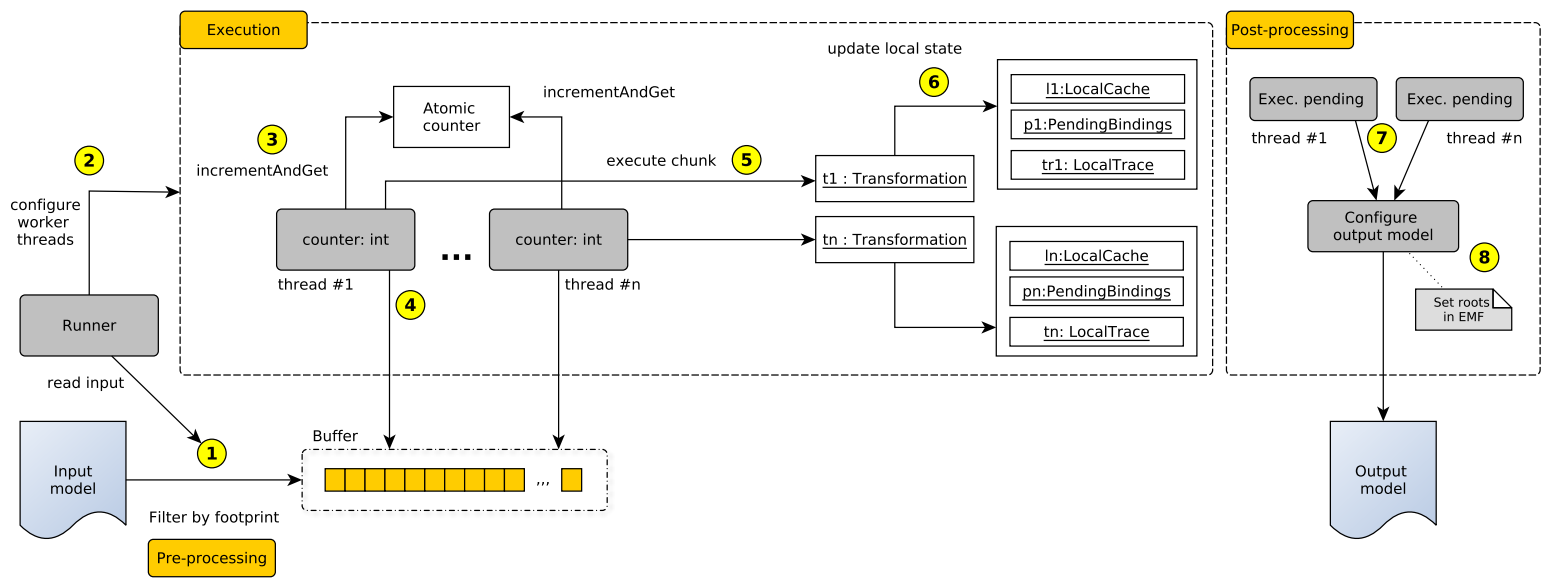

Fig. 6. Components of the technical realization of the parallel transformation algorithm.

the end, but this implies some overhead. Therefore, we have implemented a mixed strategy in which we split the buffer in two parts. The first one is statically divided into chunks of size $0.75 \times$ buffer_size/num_workers, which are large enough for each worker to start transforming elements. As soon as these chunks are finished, the algorithm continues with a dynamic scheduling strategy that uses smaller chunks (10 elements in the current configuration) to prevent workers from being idle.

Each worker uses a new instance of the transformation, given that we are using data-based parallelism, which has its own local state so that threads do not compete to access shared resources (label 6). For each element of a chunk, we try to find a matching rule. In practice, rule matching consists of checking the rules of the transformation in some order. Actually, in ATL the order is irrelevant because a given source element can only be matched by one rule, otherwise a runtime error is raised. Algorithm 2 illustrates the style of the code generated for matching the rules of the running example.

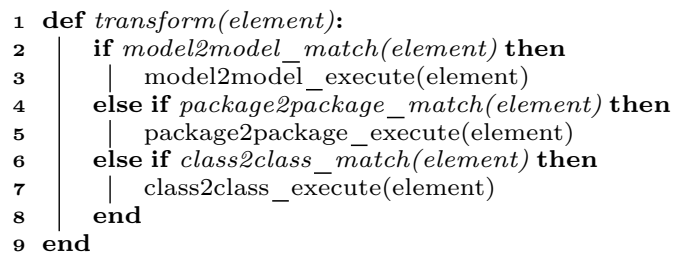

Algorithm 2: Example of rule matching

If a matching rule is found, the output elements are created and a trace record is generated to establish a mapping between the input and output elements. Thus, each transformation instance contains a partial trace (LocalTrace in the figure) to store such records locally to avoid any locking situation. In addition, the RHS of each binding is evaluated. Primitive bindings are assigned directly, but non-primitive bindings (i.e., those whose RHS evaluation returns a set of model elements) cannot be evaluated because the corresponding target models may not have been transformed yet. Thus, we delay this task by recording the fact that such dependency has yet to be resolved (PendingBindings in Fig. 66). This approach is a generalization of 20 in which special identifiers are used to construct model references which are yet to be resolved. The advantage in this case is that it is independent of the metamodelling framework and the subsequent resolution does not require to traverse the full target model, but can be done by a constant-time look up.

Post-processing. The main task of this phase is to resolve bindings, given that now all target model elements are available. For each unresolved binding its RHS is used to look up the partial traces in order to retrieve the corresponding target elements and assigning them to target features (label 7). In the sequential scenario, looking up a single trace has a constant cost $O(1)$ because the trace model can be indexed by source element using a hash map. However, an undesirable effect of the parallelization is that, now, each trace lookup has a cost proportional to the number of worker threads, because for $p$ threads we may need $p$ accesses to the partial traces (that is, the cost is $O(p)$. To mitigate this shortcoming, we also execute the post-processing in parallel. This requires classifying unresolved references into overlapping and non-overlapping. Two references are overlapping if they may potentially cause a race condition when set in parallel $]^{1}$ This is the case of opposite references and references that are set in more than one location in the transformation. In the example, client and supplier are non-overlapping, but packagedElement is overlapping because there are several assignments in the same rule. At the end of this phase, the output model may be configured as required by the underlying meta-modeling framework (label 8). In particular, if the target model is in EMF format, we need to establish the root elements of the resulting EMF resource.

This algorithm exhibits almost the same functional behavior of the original ATL algorithm, but it enables data parallelism. The only observable difference is that the order in which root elements appear in the model is not deterministic (i.e., elements which are not assigned to any containment reference) because each run may allocate chunks differently. Sect. 6 evaluates the speed up that can be obtained by applying it to exploit multi-core CPUs. However, it is possible to achieve even greater performance by optimizing the compilation of the sequential part of the transformation.

\section{Optimizations}

The availability of typing information allows our compiler to target a typed runtime environment like the JVM. This

1. Some meta-modelling frameworks, including EMF, are not thread-safe. 
already provides a performance improvement over dynamically typed languages like ATL or ETL, and over interpreterbased approaches like most QVT implementations. In addition, another significant increase in performance is possible by applying a number of optimizations to handle common transformation scenarios and idioms. The most relevant optimizations that A2L implements are described next.

\subsection{Optimizations at the transformation level}

These optimizations are intended to improve the performance of the execution of the transformation rules. They include the process of matching input elements and the binding execution.

Matched rule ordering. Our algorithm tries to match each input element against the input pattern of each matched rule. Algorithm 2 shows the generated code. The sooner the matching rule is identified, the more efficient the process is, because it avoids checking unnecessary conditions. Finding the matching rule as soon as possible depends on three main factors: the order in which the patterns are checked, the complexity of the rules' filters, and the structure and contents of the input model (some rules are matched more frequently than others depending on the particular model).

Our approach is to heuristically prioritize rules according to their filters. We count the number of OCL elements to be evaluated as part of the filter execution, and check the rules with fewer elements first. The rationale is that, in the worst case, all rules must be checked and thus it is preferable to check the cheaper ones first. If a given element is eventually matched by a rule with a costly filter, the time spent on checking the wrong rule filters first is low. However, other heuristics are possible, such as estimating the chances of matching an element by considering the operations used in the filter (e.g., equality operation would match less elements than inequalities).

Transformation footprinting. We use the transformation footprint to filter the input model in order to consider only those elements that may actually be matched by some matched rule. Notably, we only use the partial footprint, meaning that we are only interested on the types declared in the source pattern of the rules. In the running example, the partial footprint of that transformation consists of the set footprint $=\{$ Package, Class $\}$. This is specified in line 3 of Algorithm 1. We intend to significantly reduce the buffer size in scenarios in which the transformation only matches a small subset of the model. Although the default ATL algorithm behaves differently (it computes an associative table to gather objects per type) it would also benefit from this optimization since the table size could be smaller by only recording needed elements. Moreover, we use this step to pre-compute global data such as ClassDeclaration.allinstances (), thus avoiding another model traversal.

Binding handling. ATL relies on binding resolution to assign target references implicitly. To resolve a binding, ATL checks if the the value of the RHS is a primitive value, an object, or a collection of primitive values and/or objects. If it is a collection, it might be the case that it is a nested collection, in which case it needs to be flattened. A2L does not need to check these conditions at runtime since it knowns at compile time whether the RHS contains primitive or object values, and whether collections are nested or not.
Trace footprint reduction. ATL relies on recording trace links between the input elements matched by a rule and the corresponding elements created upon execution. A transformation engine without access to information about rule relationships will generate trace links even in cases where they are not needed. In A2L, we apply an optimization to reduce the trace memory footprint, namely, we analyse which rules may need to resolve a given binding. There are two main scenarios: a) if a matched rule is never used to resolve a binding, the link between the input element and the primary output pattern element does not need to be recorded, and b) if a matched rule has more than one output pattern element, there is no need to record the trace link for the secondary elements (i.e., all elements except the first one) unless there is a resolveTemp operation which retrieves them (in ATL, the resolveTemp operation is used to explicitly retrieve a target element from a given source element).

This optimization is useful to reduce the memory footprint when there are rules that create, for a single input element, a large connected set of elements, but other rules only need to link a single element - typically the parent of these elements.

\subsection{OCL-related optimizations}

As mentioned in the introduction, A2L supports all ATL and OCL datatypes including collections, maps and tuples, and the standard OCL library. ATL makes heavy use of OCL expressions for navigating the models, selecting elements in the rule filters, and for calculating the values of the target elements' features. However, the evaluation of these OCL expressions is often inefficient, in particular regarding the use of collections. This is due to the fact that OCL datatypes are immutable, which means that each operation over a collection needs to return a new collection. The transformation engine should therefore use internally a library for immutable collections, but this is sometimes not enough when dealing with very large models. The problem is that these auxiliary collections can be huge and they can be unnecessarily created several times during an OCL expression evaluation. Moreover, the typical OCL access patterns for collections are not well suited for immutable collections (e.g., in sequences including appends an element, but in an immutable list prepend is typically more efficient).

To address this issue, we have a two-step approach. In the first step, we perform escape analysis to check whether a given collection may be modified in more than one location. In this case, it is not possible to apply any optimization and we resort to immutable collections. However, if a collection is not going to be shared, we mark each involved (sub-)expression as mutable, so that in the second step the compiler is free to generate code using mutable collections. For instance, in the following listing, the first example shows a piece of ATL for which it is possible to generate code using mutable collections, whereas in the second example we must use immutable collections.

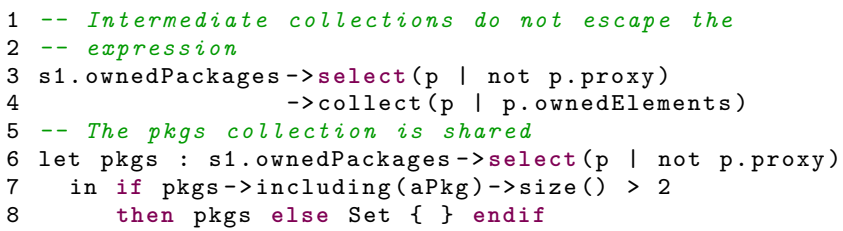


TABLE 1

Summary of the most relevant OCL optimisations. The target code is written in a Java-like pseudocode for simplicity.

\begin{tabular}{|c|c|c|}
\hline Name & Example & Generated code \\
\hline $\begin{array}{l}\text { Mutable } \\
\text { addition }\end{array}$ & $\begin{array}{l}\text { aPkg. ownedElements } \\
\quad \text {->select (plp.isProxy) } \\
\quad \text {->including (aClass) }\end{array}$ & $\begin{array}{l}\text { res }=\text { new Arraylist }<\text { AbstractTypeDcl }>() \\
\text { for }(0 \text { : aPkg.get } 0 \text { wnedElements }()) \\
\text { if (o.isProxy ()) } \\
\text { res.add(o) } \\
\text { res.add(aClass) }\end{array}$ \\
\hline $\begin{array}{l}\text { Filter and check } \\
\text { existence }\end{array}$ & $\begin{array}{l}\text { aPkg. ownedElements } \\
\text {->select (p|p.ocliskindOf (JAVA! ClassDeclaration)) } \\
\text {->exists (c|c.isAbstract) }\end{array}$ & $\begin{array}{l}\text { boolean result = false } \\
\text { for (o : aPkg.get } 0 \text { wnedElements }()) \\
\text { if (o instanceof ClassDeclaration) } \\
\text { ClassDeclaration cd = (ClassDeclaration) o } \\
\text { if (cd.isAbstract ()) } \\
\text { result = true } \\
\text { break }\end{array}$ \\
\hline Filter and count & $\begin{array}{l}\text { aClass.bodyDeclarations } \\
\text {->select (d|not d.isProxy) } \\
\text {->select (d|d.modifier.static) } \\
->\operatorname{size}()\end{array}$ & $\begin{array}{l}\text { size }=0 \\
\text { for (d : aClass.getBodyDeclarations ()) } \\
\text { if (!d.isProxy() \&\& } \\
\quad \text { d.getModifier (). isStatic ()) } \\
\quad \text { size++ }\end{array}$ \\
\hline Filter and map & $\begin{array}{l}\text { aPkg. ownedElements } \\
->\text { select (p|not p.proxy) } \\
->\operatorname{collect}(p \mid p \cdot c l a s s) \\
->\operatorname{collect}(\mathrm{c} \mid \mathrm{c} \cdot \text { name })\end{array}$ & 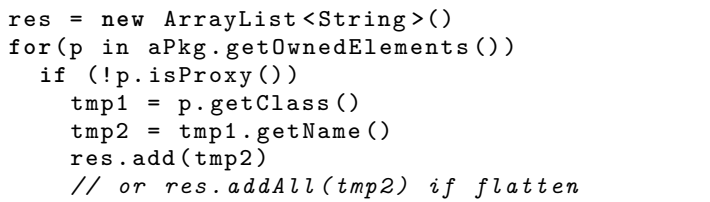 \\
\hline $\begin{array}{l}\text { Collection con- } \\
\text { version }\end{array}$ & $\begin{array}{l}\text { classes } \\
\quad->\operatorname{collect}(c \mid c \cdot \text { name }) \\
\quad>\operatorname{asset}()\end{array}$ & $\begin{array}{l}\text { // Target collection is created beforehand } \\
\text { res = new HashSet<String }>() \\
\text { for (c in classes) } \\
\quad \text { res.add (c) }\end{array}$ \\
\hline Indexing & $\begin{array}{l}\text { (1) JAVA! ClassDeclaration. allinstances() } \\
\text {->exists (p I p.name = name) } \\
\text { (2) JAVA!ClassDeclaration. allinstances () } \\
\text {->select (p I p.name = name) }\end{array}$ & $\begin{array}{l}\text { // A global object is initialized in } \\
\text { // the pre-processing phase with } \\
\text { // (1) Set<String } \\
\text { // (2) Map<String, List<ClassDeclaration>> } \\
\text { globalContext.existsIndex. contains (name) } \\
\text { globalContext. selectIndex.get (name) }\end{array}$ \\
\hline
\end{tabular}

In a second step, we use the results of the escape analysis. We try to optimize certain access patterns for which we can generate optimized code that avoids redundant creation of temporary collections by using mutable collections. For instance, the evaluation of the following OCL expression using immutable collections requires traversing two intermediate collections (one for select and another for collect) whose size is equal to the number of UML! Class instances.

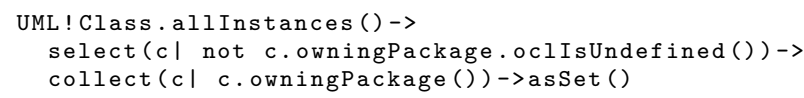

However, if the resulting collection is not shared we can generate more efficient code which avoids unnecessary processing and memory usage. Our compiler detects this particular pattern and generate specific code for it, using only one traversal of the source collection and without intermediate collections. The following listing illustrates the code that would be generated.

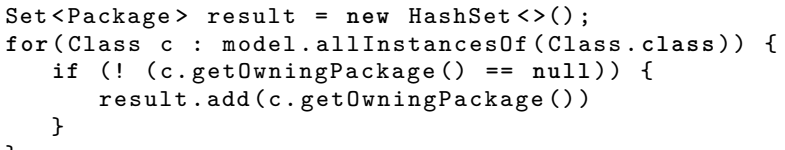

Table 1 shows a summary of the most relevant optimizations, described by means of their context, a prototypical example of each one, and how they are implemented in $\mathrm{A} 2 \mathrm{~L}$, i.e, the Java code generated for them.

\subsection{Automatic caching}

This optimization deals with OCL code that computes the same value several times, and such computation can be potentially time consuming. It is possible to increase the execution performance if such computations are cached so that they are reused in subsequent accesses. ATL supports caching by factorising code in attribute helpers, but this requires the developer to identify which code locations should be cached. Our compiler detects some of these locations and generates code that caches repeated results. In particular, we consider a hot spot a sub-expression within a nested loop such that it starts with a variable that is independent of the outermost loop. For instance, in the following code the value $c 2$.allSuperClasses ()$->$ reject (...) is reused across iterations of the outer forAll because it is cached.

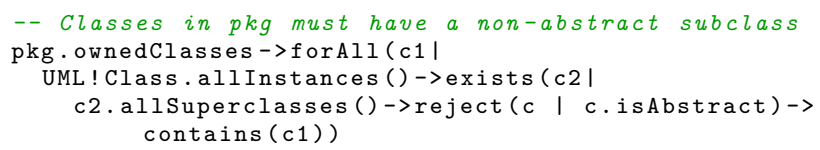

The last row of Table 1 shows another form of automatic caching, in which certain access patterns are compiled as an indexing operation. The index is filled in the pre-processing phase in order to provide fast access during the transformation execution.

\section{Validation}

To evaluate our approach, we have defined four research questions regarding the correctness of the obtained transfor- 
mation output models, the completeness of the ATL language support, the speedup compared to existing ATL engines and, finally, the scalability of A2L, i.e., the speedup gained when raising the number of available cores. To answer these questions, we carried out an empirical case study 22$]$ by following the guidelines for conducting empirical explanatory case studies by Roneson and Hörst 23. Moreover, the implementation, case studies and scripts to reproduce our results are available at http://github.com/anatlyzer/a2l.

In the next subsections, we describe our research questions and the case studies and metrics we have used to answer these questions. Finally, we discuss the answer to each research question and the overall threats to validity of our proposal.

\subsection{Research Questions}

Our study addresses the following four research questions. With these questions, we aim to justify the use of our compilation strategy and our parallelization approach in order to significantly improve the performance of ATL transformations. At the same time, we argue about the correctness and completeness of our approach.

RQ1 Compiler Correctness: Does the code generated by the A2L compiler exhibit the same functional behavior as the standard ATL engine? To validate the correctness, we compared the results of running transformations compiled with A2L against the results of running the same transformations on the standard ATL VM. We resort to an available set of regression tests already used by ATL transformation engines 13 to test their correctness.

RQ2 Compiler Completeness: How much of the ATL language is the A2L compiler able to deal with? To validate its completeness, we evaluated the coverage of the ATL language, defined by the ATL metamodel, for which the A2L compiler provides support. Moreover, we have manually checked against the ATL documentation which features are actually supported by A2L.

RQ3 Performance: What is the gain in performance when compared with ATL VM and EMFTVM? To evaluate the performance of the implementation we have used seven case studies, which exercise several transformation styles and ATL constructs. We compared the execution times of different A2L versions (non-optimized, optimized, sequential and parallel) with the standard ATL VM and EMFTVM. EMFTVM is a newer ATL engine that compiles to Java bytecode on the fly and it is reported to achieve gains of $80 \%$ in basic benchmarks. In the experiments, we used A2L in both sequential and parallel mode. The sequential execution allows us to show the gains obtained only by the use of static analysis and optimizations. The parallel execution aims to validate our parallelization strategy. Since the optimizations are optional, we also compared the executions with and without the optimizations, to assess their impact on performance.

RQ4 Parallelism: What are the effects of adding more cores? We analyse how the number of cores influences the execution times of ATL transformations parallelized by A2L. We have executed our case studies using an increasing number of threads and recorded the obtained speedups.

\subsection{Experimental Setup}

\subsubsection{Case Studies}

RQ1 and RQ2 are evaluated using the same set of 24 regression tests 13 that the ATL team used to validate the correctness of two consecutive versions of the ATL Virtual Machine. Each of these tests consists of one model transformation and all the necessary artifacts needed to execute the transformation, i.e., the input and output metamodels, and a sample input model. Since none of these tests provides large models, to answer RQ3 and RQ4, we considered seven additional case studies: java2uml (reverse engineering Java code into UML models), java2graph (creates a graph of dependencies between Java classes), dblpv1 (query the DBLP database to obtain authors and associated information), dblp2bibtex (map DBLP entries to BibTeX records), identity (copy transformation of the IMDB database), findcouples (extracts actors from IMDB who played together) and airquality (queries weather data obtained from sensors). For these transformations, we have models with up to 5.6 millions elements, and 1.2 GB when serialized and stored in disk.

In order to characterize our benchmark, we have considered six dimensions, which are used in Table 2 to summarize the main characteristics of the performance case studies:

1) I/O size is the expected size of the output model with respect to the size of the input model.

2) Matching cost is the expected cost of rule matching, in particular the complexity of the rule filters.

3) Rule cost is the expected cost of rule execution, which is the complexity of the RHS of the bindings.

4) FP size is the footprint size with respect to the input model size, that is, if the transformation has rules that attempt to match all input elements.

5) OCL refers to the dominant OCL elements in the transformation: collection intensive, usage of allinstances, conditionals, etc.

6) Finally, we count the transformation elements in order to have an indication about its "size": number of matched rules $\left(M_{R}\right)$, number or called or lazy rules $\left(\mathrm{L}_{\mathrm{R}}\right)$, number of helpers $(\mathrm{H})$, dependencies between rules as the number of bindings for references (B), number of imperative blocks (I).

All transformations except identity and dblp2bibtex generate output models which are smaller, in terms of number of elements, than their corresponding input models (I/O size). This means that they either rule out many elements in the rule filters (they have a high matching cost) or its footprint with respect to the original meta-model is small (they purposely lack rules to match certain elements). Both identity and dblp2bibtex exercise the ability of the engine to handle many binding resolutions. Regarding the matching cost, we have that airquality, dblpv1 and dblp2bibtex have at least one rule filter which traverses collections or accesses all instances of a given type, so it is expected to be costly, whereas the other four transformations have very simple or no rule filters. 
TABLE 2

Main features of the case studies.

\begin{tabular}{l|lllll|lllll} 
Name & $\mathbf{M}_{\mathbf{R}}$ & $\mathbf{L}_{\mathbf{R}}$ & $\mathbf{H}$ & $\mathbf{B}$ & $\mathbf{I}$ & $\mathbf{I} / \mathbf{O}$ size & Match. cost & Exec. cost & FP size & OCL elements \\
\hline airquality & 1 & 0 & 0 & 0 & 0 & $I_{\text {size }}>O_{\text {size }}$ & high & low & equal & collections, allInstances \\
dblpv1 & 1 & 0 & 1 & 0 & 0 & $I_{\text {size }}>O_{\text {size }}$ & medium & medium & equal & collections \\
dblp2bibtex & 7 & 2 & 1 & 1 & 0 & $I_{\text {size }}=O_{\text {size }}$ & medium & medium & equal & allinstances \\
findcouples & 3 & 1 & 2 & 5 & 2 & $I_{\text {size }}>O_{\text {size }}$ & low & high & equal & set operations \\
identity & 5 & 0 & 0 & 8 & 0 & $I_{\text {size }}=O_{\text {size }}$ & low & low & equal & property access \\
java2graph & 2 & 0 & 3 & 2 & 0 & $I_{\text {size }}>O_{\text {size }}$ & low & medium & smaller & conditionals \\
java2uml & 3 & 5 & 2 & 6 & 0 & $I_{\text {size }}>O_{\text {size }}$ & low & medium & smaller & collections
\end{tabular}

The execution of cost of airquality and dblpv1 is low because they are "query transformation" whose target elements have simple initialisations. In this respect identity also has simple initialisations, but it has more binding dependencies which makes the post-processing phase time consuming.

\subsubsection{Evaluation Metrics}

To answer our research questions, we use several metrics depending on the nature of the research question.

Model Comparison Metrics (RQ1): To evaluate research question RQ1, correctness, we need to compare the resulting models after running the code produced by the A2L compiler, with those obtained from the execution of the standard ATL VM. For this, we used EMF Compare, a model comparison framework that compares two models and reports differences between them, such as additions, deletions, and updated elements.

Language Coverage Metrics (RQ2): To evaluate RQ2, we computed the footprints of the ATL transformations with respect to the ATL metamodel. This gives an estimation of how many features are tested by the test cases.

Execution Performance Metrics (RQ3 and RQ4): To evaluate research questions RQ3 and RQ4, we calculated the execution time of the seven case studies listed in Table 2 using a large model as input. We run the experiments on a desktop machine with Ubuntu 18.04 and kernel 5.3.0, a i7$5820 \mathrm{~K} \mathrm{CPU}$, with 6 cores at $3.30 \mathrm{GHz}$ and hyper-threading (12 threads) and 16 GB of RAM, which is expected to be representative of a typical setup of a professional developer. We have used Java 8 (OpenJDK 1.8.0_252) configured with the default options except for the heap size which was set to 8 GB (-Xms=8196m -Xmx=8196m), except for dblp2bibtex which was set to $12 \mathrm{~GB}$. Each case study is run 10 times with the different engines, discarding the first two runs (as warm up). We perform the 10 executions together in the same VM instance, but after each execution we wait until the garbage collector has released the used memory. We report the average results.

\subsection{Result Analysis}

\subsubsection{Results for RQ1}

We executed the 24 regression test cases and compared the output models produced by the standard ATL engine and by A2L. All test cases produced the same results, apart from five of them that could not be directly executed.

We could not compile the ATL2Problem transformation with A2L because its typing is too convoluted for ANATLYZER and it cannot properly infer the type of a couple of expressions. Another two unsupported transformations were $D S L 2 X M L$ and KM32DSL, because they set global variables using some reflective operations which are currently unsupported by A2L. Similarly, the SpreadsheetMLSimplified2Trace transformation uses global attributes in a way that adds serious performance penalties to the parallel algorithm. It is worth noting that all of these four transformations could be rewritten so that they could be compiled by A2L. For instance, when there are global variables involved, a strategy could be to use helpers to compute the global information from the source model each time (possibly with some penalty in the execution time). When the problem is related to typing, it might be possible to insert specific annotations (e.g., a dummy version of oclAsType) in dedicated places to guide the type inference performed by ANATLyzer [24]. Finally, the ATL and A2L output models of the XML2DSLModel transformation were slightly different because this transformation suffers from child stealing, i.e., an element is set to a containment reference more than once.

\subsubsection{Results for RQ2}

The test cases used in our experiments cover $88 \%$ of the ATL meta-model. The missing $12 \%$ belongs to meta-model elements used to represent declaration of libraries and query modules, unique lazy rules, entry point rules, rule inheritance and map types. We have separate tests for all of these elements, except query modules and rule inheritance which are currently not supported. Although we do not foresee any difficulty in supporting rule inheritance in the future, we decided not to implement it at this stage because it is rarely used in practice 25 .

We have used the ATL manual as reference for our implementation. Table 3 shows the language features covered by A2L. We support all forms of matched rules (i.e., 1:1, 1:N, N:1 and $\mathrm{N}: \mathrm{N}$ ) and all major ATL features.

\subsubsection{Results for RQ3}

Table 4 shows the execution times of the case studies in our desktop machine. It compares two versions of the ATL engine (the standard VM and EMFTVM) against four different configurations of A2L: sequential mode without optimizations (seq $\mathrm{O}-$ ), sequential mode with optimizations (seq $\mathrm{O}+$ ), parallel (using 12 cores) without optimizations enabled (par $\mathrm{O}-$ ), and parallel (using 12 cores) with optimizations (par $\mathrm{O}+)$ - or simply A2L, since this is the default A2L mode. The figures shown in Table 4 correspond to the average execution time in seconds of eight runs of each transformation. Note that the execution time excludes model loading, but in the case of A2L, it includes the three phases of the algorithm: pre-processing (filling the buffer after model loading), transformation execution, and post-processing. We did not include the parallel ATL 9] implementation (pATL) because it is not supported anymore and we could not make it work reliably. 
TABLE 3

ATL coverage.

\begin{tabular}{lcl}
\hline Feature & Support & Observations \\
\hline Matched rules & Yes & \\
Input elements $=1$ & Yes & \\
Input elements $>1$ & Yes & Via rewriting \\
Output elements $=1$ & Yes & \\
Output elements $>1$ & Yes & \\
Rule inheritance & No & \\
Binding resolution & Yes & \\
resolveTemp & Yes & Secondary elements resolution \\
Lazy rules & Yes & \\
Unique lazy rules & Yes & \\
Called rules & Yes & Also end/entry point rules \\
Helpers & Yes & Context and global helpers \\
OCL & & \\
Collection types & Yes & \\
Tuple types & Yes & \\
Collection iterators & Yes & \\
Iterate operation & Yes & \\
Reflective operations & No & \\
\hline
\end{tabular}

Table 5 shows the speedups obtained by the different transformation engines and A2L execution modes. For each one, we calculated the individual speedups achieved in all the case studies (excluding the dblp2bibtex model transformation in the comparisons between ATL/EMFTVM and A2L because it could not be executed in ATL and EMFTVM). Every cell shows a tuple with the minimum (left) and maximum (right) values, as well as its geometric mean (center), which is the most informative way to represent the average speedup as expected by users 26]. The standard ATL engine is consistently slower than the other options. This is due to its sub-optimal implementation of immutable collections which hinders its ability to handle scenarios with extensive processing of large collections [21]. In particular, the airquality test case heavily exercises this feature and shows that EMFTVM is far more efficient (50 vs. 1,280 secs). This is because it uses a custom implementation of immutable (and lazy) collections. Moreover, EMFTVM compiles to JVM bytecode on the fly, which provides additional gains. These two features combined makes it normally much faster than the standard ATL engine (except for the findcouples example).

A2L obtains significant gains over both engines in all execution modes. Next, we only discuss improvements w.r.t. EMFTVM since they also imply gains over standard ATL.

In the slowest A2L execution mode (A2L seq $\mathrm{O}-$ ), the speedup w.r.t. EMFTVM is between $1.37 \mathrm{x}$ and $10.4 \mathrm{x}$ depending on the application. Given that both A2L and EMFTVM target JVM bytecode, this improvement can be explained (in addition to differences in the engine internals) by the fact that A2L does not need to generate code for dynamic checks and model accesses. Table 4 shows that all case studies benefit from this improvement. The findcouples case study is the slowest, with a relatively modest performance gain of $1.37 \mathrm{x}$. The main bottleneck is a nested loop which computes the same value in different rule applications. This shortcoming is addressed by our optimizer through automatic caching. Given these results, it can be stated that it is possible to have a significant performance improvement by changing the design of the transformation language from dynamically to statically typed. All other A2L execution modes (with optimizations and in parallel) outperform EMFTVM even more, with speedups that range between $2.32 \mathrm{x}$ and $38.28 \mathrm{x}$ (A2L seq $\mathrm{O}+$ ), 1.79x and 21.43x (A2L par $\mathrm{O}-$ ), and even between $2.4 \mathrm{x}$ and $245.83 \mathrm{x}$ in the case of the A2L default behavior (A2L par $\mathrm{O}+$ ), depending on the test case. The geometric means indicate expected average speedups of A2L against EMFTVM of $3.37,8.59,6.49$ and 22.42 , respectively.

We also wanted to investigate the individual effects of optimization and parallelization in A2L. This is why we compared the performance of the four possible execution modes of A2L: A2L seq $\mathrm{O}-$, A2L seq $\mathrm{O}+$, A2L par $\mathrm{O}-$, and $\mathrm{A} 2 \mathrm{~L}$ par $\mathrm{O}+$.

Using as baseline the sequential mode without optimizations (seq $\mathrm{O}-$ ), the speedup obtained by enabling optimizations (seq $\mathrm{O}+$ ) ranges between $0.84 \mathrm{x}$ and $66.76 \mathrm{x}$ depending on the case study. The worse case is the identity application, in which the execution time even increases. This is because the partial footprint of the transformation is equal to the source meta-model, and therefore our footprint filtering optimisation only adds overhead to the optimised version but does not reduce the buffer size (there are no other optimisations in that transformation). Therefore, when the partial footprint is equal to the meta-model, it is better not to activate this optimisation. The best case occurs in the dblp2bibtex transformation, where optimizations achieve a speedup of $66.76 \mathrm{x}$ due to the automatic indexing optimization. The speedup obtained by the use of optimizations in the airquality case study is $5.2 \mathrm{x}$, because this transformation is particularly well-suited for collection optimizations given that it applies several OCL iterators to the set of objects returned by allinstances (i.e., it needs to traverse the complete model). The findcouples case study gets a speedup of $3.84 \mathrm{x}$ thanks to the automatic caching optimization. The rest of the transformations obtain speedups of $1.64 \mathrm{x}, 6.31 \mathrm{x}$ and $1.65 \mathrm{x}$, respectively, when optimizations are enabled, with a geometric average of $4.07 \mathrm{x}$.

To analyse the effects of parallelization on the performance of A2L (using the 12 threads in our experimental desktop machine), we compared our baseline A2L seq $\mathrm{O}$ - with A2L par $\mathrm{O}-$. The resulting speedup ranges between $1.15 \mathrm{x}$ and $4.66 \mathrm{x}$, which represents a significant improvement, although not as noticeable as the one obtained with the optimizations. This is clear in the comparison between optimizations (seq $\mathrm{O}+$ ) and parallelization (par $\mathrm{O}-$ ), where the former outperforms the latter $2.14 \mathrm{x}(\simeq 1.0 / 0.47 \mathrm{x})$ on average.

The speedup obtained by combining optimizations and parallelization ranges between $1.11 \mathrm{x}$ and $92.02 \mathrm{x}$ depending on the case study, compared to the sequential baseline (seq $\mathrm{O}-$ ). Again, the identity transformation gets the smallest improvement (1.11x) while dblp2bibtex obtains the largest gain $(92.02 \mathrm{x})$. The rest of the test cases achieve speedups of $32.23 \mathrm{x}, 22.66 \mathrm{x}, 1.70 \mathrm{x}, 14.19$, and $4.47 \mathrm{x}$, respectively.

Finally, if we compare the execution times of the two parallel modes (with and without optimizations), the one with optimizations obtain speedups that range between $0.96 \mathrm{x}$ (identity) and 53.48x (dblp2bibtex). This means that optimizations also have a positive effect in the parallel results, mainly because they reduce memory usage, thus reducing the pressure over the garbage collector (i.e., the less garbage collection pauses the better, because a pause stops all threads and causes an important degradation in the speedup).

In summary, A2L achieves significant speedups compared to ATL and EMFTVM. By combining both optimizations and parallel execution, A2L is able to outperform EMFTVM 
TABLE 4

Performance comparison. Execution on an i7-5820K CPU@3.30GHz - 6 cores (12 threads). Time in seconds.

\begin{tabular}{lrrrrrrr}
\hline & $\begin{array}{r}\text { \#elements } \\
(\text { millions })\end{array}$ & ATL & EMFTVM & $\begin{array}{r}\text { A2L: seq O- } \\
(1 \text { thread })\end{array}$ & $\begin{array}{r}\text { A2L: seq O+ } \\
(1 \text { thread })\end{array}$ & $\begin{array}{r}\text { A2L: par O- } \\
(12 \text { threads })\end{array}$ & $\begin{array}{r}\text { A2L: par O+ } \\
(12 \text { threads })\end{array}$ \\
\hline airquality & $0.1 \mathrm{M}$ & 1280.40 & 50.70 & 6.65 & 1.32 & 3.39 & 0.21 \\
findcouples & $3.5 \mathrm{M}$ & 1765.09 & 1908.89 & 1395.66 & 363.28 & 299.54 & 61.60 \\
identity & $3.5 \mathrm{M}$ & 269.00 & 71.73 & 11.63 & 13.87 & 10.08 & 10.48 \\
java2graph & $4.4 \mathrm{M}$ & 146.33 & 2.32 & 1.65 & 1.00 & 1.30 & 0.97 \\
java2uml & $4.4 \mathrm{M}$ & 49.18 & 42.05 & 27.28 & 4.32 & 14.59 & 1.92 \\
dblpv1 & $5.6 \mathrm{M}$ & 75.66 & 48.68 & 4.68 & 2.84 & 2.27 & 1.05 \\
dblp2bibtex & $5.6 \mathrm{M}$ & - & - & 783.07 & 11.73 & 455.15 & 8.51 \\
\hline
\end{tabular}

TABLE 5

Speedups achieved between the transformation engines: ATL, EMFTVM and the different A2L options.

\begin{tabular}{|c|c|c|c|c|c|}
\hline Speedups & EMFTVM & $\mathrm{A} 2 \mathrm{~L}$ seq $\mathrm{O}-$ & $\mathrm{A} 2 \mathrm{~L}$ seq $\mathrm{O}+$ & $\mathrm{A} 2 \mathrm{~L}$ par $\mathrm{O}-$ & $\mathrm{A} 2 \mathrm{~L}$ par $\mathrm{O}+$ \\
\hline ATL & {$[0.92 ; 4.64 ; 63.06]$} & {$[1.26 ; 15.63 ; 192.6]$} & {$[4.86 ; 39.90 ; 966.63]$} & {$[3.37 ; 30.15 ; 377.25]$} & {$[25.58 ; 104.14 ; 6207.98]$} \\
\hline EMFTVM & {$[0.00,1.0000]$} & {$[1.37 ; \mathbf{3 . 3 7} ; 10.4]$} & {$[2.32 ; \mathbf{8 . 5 9} ; 38.28]$} & {$[1.79 ; \mathbf{6 . 4 9} ; 21.43]$} & {$[2.40 ; \mathbf{2 2 . 4 2} ; 245.83]$} \\
\hline A2L: seq, $\mathrm{O}-$ & & 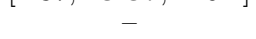 & {$[0.84 ; \mathbf{4 . 0 7} ; 66.76]$} & {$[1.15 ; 1.90 ; 4.66]$} & 9.69 \\
\hline A2L. seq, O+ & & & - & {$[0.03 ; \mathbf{0 . 4 7}$} & 2.38 \\
\hline A2L: par, $\mathrm{O}-$ & & & & - & 5.11; \\
\hline
\end{tabular}

between $2.4 \mathrm{x}$ and $245.83 \mathrm{x}$, with an expected average of $22.42 \mathrm{x}$. In this way, A2L execution times for large models become acceptable in all cases, which is an indication that ATL can become a competitive model transformation language when compiled with A2L. In addition, A2L is capable of running transformations such as dblp2bibtex that may not be executed with the other engines because of the excessive usage of collection operations over very large collections. In practice, these results also mean a much better developer experience because the transformations can be used as part of the development process without incurring long waiting times.

\subsubsection{Results for RQ4}

To answer this question, we have executed the case studies using an increasing number of cores, from 1 to 12 . Our algorithm has three phases: pre-processing, execution and postprocessing, but only the execution phase is expected to have a speedup due to parallelism, because the parallel execution of the post-processing phase only amortizes the cost of accessing the partial traces created by each transformation instance. The speedups obtained in the execution phase for each case study are shown in Fig. 7. We can see how two of the test cases (airquality and findcouples) scale up very well, close to the theoretical limit, which is $6 \mathrm{x}$ with 6 threads. Then, $d b l p v 1$ and identity also scale well, with speedups around $4.5 \mathrm{x}$ with 6 threads. However, the other three stop scaling soon. This can be caused by a variety of reasons. On the one hand, the rules of these transformations have a modest computation cost which may increase the competition for obtaining the next chunk from the scheduler. On the other hand, parallel computations are highly sensitive to external factors. For instance, a stop-the-world execution of the Java GC would provoke an important decrease in the speedup. Also, in Java and EMF in particular there is little control over the memory layout which may cause an increase in cache misses.

A related question is what is the scalability when taking into account all the phases of the algorithm, or in other words, how much the pre-processing and post-processing phases limit the parallel speedup. Table 6 shows the breakdown of the execution times of the case studies in sequential and in parallel

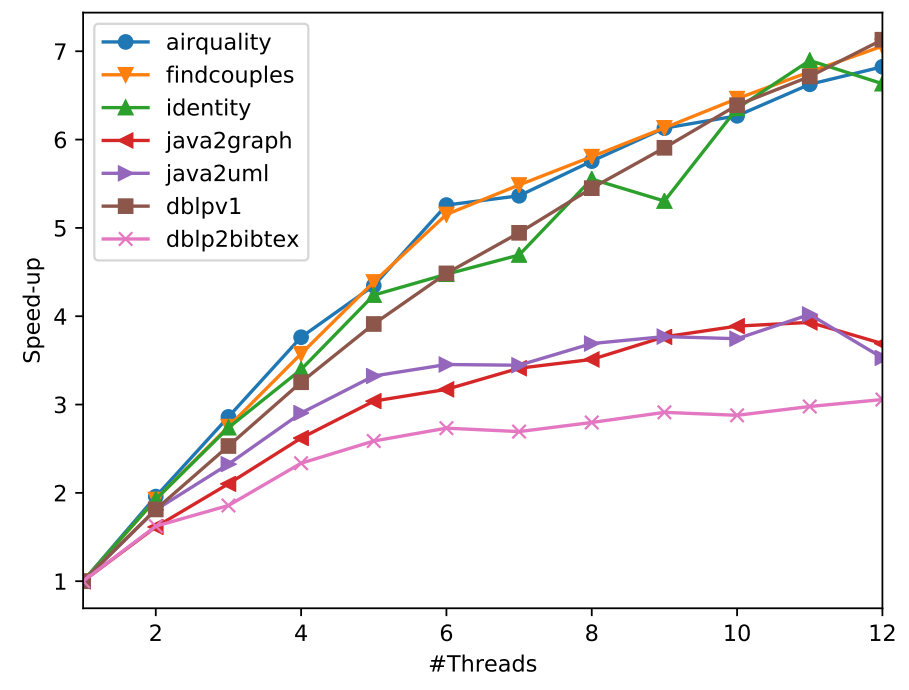

Fig. 7. Speedup of the execution phase of the transformations.

mode with 6 threads. In some transformations, like airquality, java2graph, java2uml and dblpv1, the post-processing time is negligible because they do not resolve many bindings. However, transformations with more bindings or whose bindings have many objects in the RHS, incur in large postprocessing times. Therefore, the post-processing phase represents the main bottleneck for scalability in case there are many dependencies among the elements in the rules, because the traces are distributed in the threads and there is a $O$ (numThreads) cost to access them.

Overall, it can be claimed that our algorithm exhibits good scalability, particularly for transformations whose rules have a high computational cost, but relatively few rule dependencies.

\subsubsection{Further findings}

Compatibility with ATL. The fact that ATL is a dynamic language poses the challenge of correctly inferring type information for its compilation to a typed target language such as Java. In practice this means that we require the transformation to be considered well-typed by AnATLYZER. However, 
TABLE 6

Execution times for $\mathrm{A} 2 \mathrm{~L} \mathrm{O}+$.

\begin{tabular}{lrrrrrr}
\hline & \multicolumn{3}{c}{ Sequential } & \multicolumn{3}{c}{ Par. 6 threads } \\
& Pre. & Exec. & Post. & Pre & Exec. & Post. \\
\hline airquality & 0.01 & 1.31 & 0.00 & 0.01 & 0.25 & 0.00 \\
findcouples & 0.60 & 353.65 & 9.03 & 0.61 & 68.64 & 8.48 \\
identity & 0.66 & 3.88 & 9.33 & 0.65 & 0.87 & 9.14 \\
java2graph & 0.94 & 0.05 & 0.01 & 0.93 & 0.01 & 0.01 \\
java2uml & 1.10 & 3.13 & 0.08 & 1.06 & 0.91 & 0.07 \\
dblpv1 & 0.73 & 2.11 & 0.00 & 0.74 & 0.47 & 0.00 \\
dblp2bibtex & 1.88 & 4.70 & 5.15 & 1.84 & 1.72 & 5.20 \\
\hline
\end{tabular}

when evaluating RQ1, we already found that ATL2Problem and those transformations using reflective operations could not be compiled. The fact that we have been able to compile the rest of the test cases make us believe that this is a minor issue. Besides, it is always possible to write an equivalent program compatible with A2L.

At the execution level, there might be differences in the order in which the root elements appear in the serialized model, notably when executed in parallel mode. Nevertheless, this is not a real incompatibility since this order is not prescribed by the ATL manual. There are also some differences in the way errors are handled. In particular, ATL signals rule conflicts with a runtime exception, whereas A2L ignores them and relies on ANATLYZER for signalling them at compile time.

Additional optimizations. In the experiments, we have observed that the post-processing phase is sometimes a bottleneck when a transformation needs to initialize many target references. It would be interesting to find out ways to reduce its impact. At the pre-processing level, it would be possible to filter the input model at loading time using approaches like partial model loading [27]. This would enable the engine to start the transformation without waiting for the model to be fully loaded. Regarding the evaluation of OCL expressions, more specific optimizations for common access patterns can be developed. A2L is prepared for this with a dedicated extension mechanism.

Using GPLs vs. ATL. There is a recent trend in the modeling community to use general purpose programming languages (GPL), such as Java or Scala, to develop and execute model transformations. There are several reasons that justify such a decision. For example, (a) IDE features available for GPLs such as live error reporting, quick fixes and debugging have been traditionally missing for transformation languages like ATL; $(b)$ the performance and scalability of ATL, when compared with those of GPL, are much worse; $(c)$ ATL can only deal with EMF models, which is not the format in which many models are stored (for instance, in biology or automotive applications), being rather inefficient, too. The declarative nature of ATL for specifying the transformations and the facility to navigate models using OCL was at the beginning a strong point against Java, but this is not the case any more after Java 8 supported lambda expressions. Scala was also strong in this respect, and its efficient performance has made it a good recent replacement for ATL, too.

However, our goal with $\mathrm{A} 2 \mathrm{~L}$ is to improve the situation for model transformation languages in general, and ATL in particular, showing that our design can make MT languages competitive again against these GPLs. First, the AnATLYZER tool provides very helpful and useful analyses of the ATL code which are not possible if the transformation is written in a GPL. This, together with the quick-fixing and visualization capabilities that ANATLYZER provides [11, [12 help solving the first shortcoming. Second, we have seen how the performance and scalability of the A2L generated code can be quite acceptable for transforming large models, with competitive execution times. Furthermore, the specific optimizations of A2L for the ATL code and the OCL expressions can provide interesting advantages over GPLs since navigation code could be written in the most readable manner without impact in its performance. A transformation engine also hides the technological complexity related to the modelling platforms. For instance, we internally optimize certain type of accesses to multi-valued features for which we discovered poor performance of the default EMF's internal iterators ${ }^{2}$ Moreover, the declarative nature of ATL enables the automatic parallelization of the transformation code. If a transformation is written in a GPL, this improvement has to be done manually, which is typically difficult, cumbersome, and error prone.

\subsection{Threats to Validity}

In this section, we cover the four basic types of validity threats that can affect the validity of our study 28 .

\subsubsection{Conclusion Validity}

Conclusion validity affects the ability to draw correct conclusion about the relations between the treatment and the outcome, i.e., how reasonable the conclusion is. Examples that influence this threat to validity include the choice of sample size, and the measurement of the experiment. In this respect, the correctness and coverage of the compiler have been assessed using the standard regression test suite for ATL 13. The size of this suite is relatively low, but it covers a large part of the ATL language. Regarding the performance evaluation, there might be specific transformations for which A2L performs worse than ATL and EMFTVM. To mitigate this threat, we have used transformations which exercise different types of scenarios and its analysis shows that they cover different transformation styles. Moreover, the large improvents obtained with A2L makes us confident that improvements will be achieved even in unforeseen scenarios.

\subsubsection{Construct Validity}

Construct validity refers to the extent to which the experiment setting reflects the theory, i.e., whether the research/tests are well-constructed using established standards and methods. For example, whether the type of samples are representative of the population or not; or whether the number of classes taken reflects common experience. Again, by using a standard test suite for ATL, we aimed at minimizing this threat, since the transformations that comprise the suite provide a representative subset of all kinds of transformations that can be written with ATL. Likewise, the seven additional transformations were carefully selected so that they contain the main features that can have impact on the performance and scalability of the results, and therefore can constitute a representative

2. This happens because EListIterator.hasNext() invokes List.size() instead of having its own size property, which prevents JVM optimizations 
sample of the kinds of ATL transformations in which we are interested. Moreover, the sizes of the input models were also selected according to the model sizes used in similar tests [9], 20 in order to extract comparable conclusions.

\subsubsection{External Validity}

This kind of threat limits the ability to generalize the results beyond the experiment context. In this respect, the fact that we have used representative model transformations gives us some confidence that the performance results can be generalized to the rest of the ATL model transformations. Regarding how our results could be applicable to other languages, we believe that for model-to-model rule-based languages like ETL and RubyTL, the applicability would be straightforward, provided that an appropriate type checker is available. QVTOperational is also rule-based but the rule structure is more explicit, which implies that there might be less opportunities for data-based parallelism at the matched rule level as we do. Nevertheless, the OCL compiler and optimizer could be adapted. In particular, applying these ideas to QVTOperational is part of our future work.

Moreover, we have used differently sized input models for our study to cover diverse scenarios. However, there may be scenarios where even larger input models are required to be processed by model transformations. We cannot generalize our results for very large models (going beyond 10 millions of model elements such as present in the Train Benchmark 29] for continuous model queries) based on our performed study and leave this as subject to future work.

\subsubsection{Internal Validity}

Internal validity checks whether the test or instrument measures what it is supposed to. This threat can affect the independent variable with respect to causality. That is, the results may indicate a causal relationship, although there is none. In this respect, the optimizations at the transformation and OCL expression level, as well as the parallelization algorithm used to execute the transformation have proved to be effective to significantly improve its performance. The speedup results obtained independently for each separated feature seem to corroborate our hypotheses. The compiler was designed so that these features could be independently enabled or disabled, precisely to facilitate these kinds of analyses.

\section{Related Work}

With respect to the contribution of this paper, namely the efficient execution of model transformations by data parallelism and optimizations based on static analysis information, we identify three lines of related work. First, we discuss general approaches for speeding up model transformations which apply smart execution strategies for different contexts. Second, we discuss research dedicated to the evaluation of model transformation engines. Third, we discuss approaches focusing on the parallel execution of model transformations.

\subsection{Model transformation execution strategies}

In this paper, we have investigated the creation of output models from input models by following the classical batch transformation strategy, i.e., the transformation run is considered as a fresh one which is creating a new output model from scratch for a given input model 30. In addition to this strategy, there are several other strategies for executing model transformations in particular contexts. First, incremental transformations 31-33 have been proposed for cases where output models are already available from previous transformation runs. In such cases, the transformation engine may only propagate the changes of the input models to the existing output models. Second, lazy transformations 34 have been proposed for cases where only subsets of output models are needed in a first step. In such cases, the transformation engine only creates these subsets in a first phase, while other elements are created just-in-time when they are requested. Third, streaming transformations 35] have been proposed for transforming so-called streaming models, i.e., models are considered as an open and continuous stream of model elements opposed to a fixed set of elements given at once in a closed input model. Fourth, patch transformations [36] are used in cases where transformations are evolving and the existing output models have to be migrated to newer versions of the transformations.

All of the mentioned execution strategies mainly focus on not having to re-execute the whole transformation by providing some kind of reactivity, e.g., to changes in the input models and model transformations or read access to the output models. Needless to say, identifying the transformation statements that are unnecessary to be re-executed is probably the best optimization for running transformations outside the classical batch transformation area.

While we have focused on batch transformations in this work, we do not see any major obstacle to adopt the presented optimizations also for other kind of transformation execution strategies used for the discussed contexts. On the contrary, we see the introduction and evaluation of the presented optimization techniques for these additional transformation strategies as an interesting line for future research.

In addition to the already mentioned model transformation execution strategies, in recent years there have been also major efforts in optimizing the execution of full batch transformations by following the distributed execution paradigm for transforming large models which are fragmented over different computing nodes. For instance, MapReduce has been exploited for running ATL transformations on distributed models 9. Camargo et al. presented a data-centric famework for distributed model transformations reusing the Bloom platform 37. In one of our previous work, we have employed Linda-based tuple spaces to run distributed model transformations 38. Finally, graph queries (comparable to the matching part of model transformation rules such as the in-pattern of ATL rules) are executed for large models in a distributed manner by combining incremental graph search techniques and cloud computing technologies 39 as well as by providing dedicated optimization concepts such as node sharing for efficiently executing partially redundant code fragments 40 .

In this paper, we did not consider the distribution aspect of models but rather focused on supporting the scenario of running model transformations directly on developer-scale machines having the models stored in-memory. Thus, we consider investigations on the distribution aspect in combination with our presented optimization techniques as a subject for future work. 


\subsection{Performance evaluations of transformation engines}

There is work on evaluating the performance of state-of-theart model transformation engines. For instance, Amstel et al. 41] compared the runtime performance of transformations written in ATL and in QVT, finding that the standard ATL engine outperforms the available QVT engines. From these works we can conclude that A2L may also outperform existing QVT engines, although this needs to be confirmed with the more recent versions of the QVT engines. In 42], several implementation variants of the ATL language, e.g., using either imperative constructs or declarative constructs, of the same transformation scenario have been considered and their different runtime performance was compared. However, both mentioned works [41, 42] only consider the traditional sequential execution engines.

A performance evaluation of the standard ATL engines with respect to running the transformations within database technologies is performed in 43 . In our work, we currently do not use a dedicated database technology for storing the models to be transformed. However, the presented optimization techniques for ATL rules and OCL expressions based on static analysis information may also help in cases where dedicated database technologies are used to produce even more efficient code that is subsequently executed inside the databases. An interesting future work line in this respect is to study the combination of the presented approach of this paper with current advances achieved for graph databases (cf. 44 for a survey) as well as investigations of data-parallel operations inside databases (cf. 45 for a comparison of different execution models) to allow even more efficient transformations of very large models.

Finally, we also like to mention the Transformation Tool Contest (TTC) ${ }^{3}$ that is now running for 13 years. Every year, there are dedicated transformation cases developed and submitted by the community for the community. In previous years, there have also been some transformation cases that focused on performance and scalability of model transformations. In particular, worth to mention are the Train Benchmark case, the Movie Database case and the Program Comprehension case. We have reused and partially adapted the Movie Database case and the Program Comprehension case for our evaluation, as they represent outplace batch transformations that we are considering in this paper.

\subsection{Parallel model transformations}

In recent years, there is an increased interest in parallelizing different types of model transformations which resulted in dedicated extensions for graph transformation languages, model management languages based on Epsilon, and also ATL which we discuss in the following.

First, there is work in the field of graph transformations where multi-core platforms are used for parallel execution of graph transformation rules [46], 47]. In these papers, specific focus is put on the parallel execution of the matching phase of the left-hand sides of graph transformation rules, which is considered to be the most expensive part. Another work exploits the Bulk Synchronous Parallel model for executing transformations based on the Henshin graph transformation

3. https://www.transformation-tool-contest.eu framework 48. To make use of the Bulk Synchronous Parallel model, the Henshin graph transformation rules are compiled to Apache Giraph.

Secondly, efforts have been made to speed up different types of model management programs (which can be considered as specific kinds of model transformations) for the Epsilon framework [19], [49 available in Eclipse. In particular, parallel execution support for the different model management languages provided by Epsilon has been presented in [50, 51. For instance, the authors provide data and rule parallelization approaches for the Epsilon Validation Language (EVL). However, the use of static analysis information for automatic performance improvement is only mentioned as future work.

Thirdly, there have been pioneering approaches for the parallel execution of ATL transformations. In previous work, we have presented LinTra 20, an approach for running ATL transformations on Linda-based platforms following the data-based parallelization approach. Tisi et al. 10 presented another approach for the parallel execution of ATL transformations, using a task-based approach for parallelization, as already mentioned in Sect. 2.3 .

\subsection{Synopsis}

While there are several approaches available for speeding up model transformations, the scalability of model transformations is still considered as a major challenge in MDE 52. We are not aware of any approach that uses the information from static analyses to find improved (parallel) execution strategies for model transformations. Thus, we are confident that in this paper we provide an important cornerstone for scalable and high-performance execution of model transformations, which may also help to improve other model transformation engines beyond ATL. This line of research is considered critical to the long-term success of model transformation tools, as a recent survey has revealed 30 .

\section{Conclusions}

This paper has presented A2L, a compiler for the ATL model transformation language that aims at achieving efficient transformations of large models. Improved performance and scalability is accomplished by two of the main features of A2L: the use of static information to achieve effective optimizations on both rule execution and the evaluation of the OCL expressions; and the use of data parallelism in the algorithm that implements and executes the transformation. The results show that A2L produces large performance gains with respect to existing ATL engines in both sequential and parallel modes. The figures presented in this paper should be a baseline for the expected performance of future transformation languages.

Our work can be extended along different lines of research. First, further optimizations can be pursued, mainly for domain-specific transformations where the semantics of the particular domains can be taken into account. Second, we plan to study how our work can be generalised to other model transformation languages, notably QVT-Operational. To this end we aim at designing an intermediate representation from which it is easy to reuse most of the infrastructure. Implementation-wise, it would be interesting to create an intermediate representation in which it is easier to perform 
rewritings and make our optimizations composable. Handling models from different modeling platforms, beyond EMF, or even stored as plain Java objects, is another research line we are also working on, with the goal of widening the usability of A2L. This line of research may also require the development of a dedicated debugger which allows the observation and control of the executing transformations directly on the ATL code level 53 . Finally, we would like to explore the possibilities of creating a streaming transformation engine on top of A2L as well as of employing emerging database technologies for executing our transformations for very large models.

\section{Acknowledgments}

This work was partially funded by Spanish Research Projects PGC2018-094905-B-I00, TIN2015-73968-JIN (AEI/FEDER/UE), a Ramón y Cajal 2017 research grant, and TIN2016-75944-R. Furthermore, this work was partially supported and funded by the Austrian Federal Ministry for Digital and Economic Affairs, the National Foundation for Research, Technology and Development, and by the FWF under the Grant Numbers P28519-N31 and P30525-N31.

\section{References}

[1] M. Brambilla, J. Cabot, and M. Wimmer, Model-Driven Software Engineering in Practice, 2nd ed. Morgan \& Claypool Pub., 2017.

[2] B. Selic, "What will it take? A view on adoption of model-based methods in practice," Software and System Modeling, vol. 11, no. 4, pp. 513-526, 2012.

[3] D. S. Kolovos, L. M. Rose, N. Matragkas, R. F. Paige, E. Guerra, J. Sánchez Cuadrado, J. De Lara, I. Ráth, D. Varró, M. Tisi, and J. Cabot, "A research roadmap towards achieving scalability in model driven engineering," in Proc. of the Workshop on Scalability in Model Driven Engineering (BigMDE 2013). ACM, 2013, pp. 2:1-2:10.

[4] E. Guerra, J. de Lara, D. S. Kolovos, R. F. Paige, and O. M. dos Santos, "Engineering model transformations with transML," Software \& Systems Modeling, vol. 12, no. 3, pp. 555-577, 2013.

[5] F. Jouault, F. Allilaire, J. Bézivin, and I. Kurtev, "ATL: A model transformation tool," Science of Computer Programming, vol. 72, no. 1-2, pp. 31-39, 2008.

[6] OMG, MOF QVT Final Adopted Specification, Object Management Group, 2005, OMG doc. ptc/05-11-01.

[7] L. Burgueño, J. Cabot, and S. Gérard, "The future of model transformation languages: An open community discussion," Journal of Object Technology, vol. 18, no. 3, pp. 7:1-11, Jul. 2019.

[8] J. S. Cuadrado, E. Guerra, and J. de Lara, "Static analysis of model transformations," IEEE Trans. Software Eng., vol. 43, no. 9, pp. 868-897, 2017.

[9] A. Benelallam, A. Gómez, M. Tisi, and J. Cabot, "Distributing relational model transformation on MapReduce," Journal of Systems and Software, vol. 142, pp. 1-20, 2018.

[10] M. Tisi, S. M. Pérez, and H. Choura, "Parallel Execution of ATL Transformation Rules," in Proc. of MoDELS 2013, ser. LNCS, vol. 8107. Springer, 2013, pp. 656-672.

[11] J. S. Cuadrado, E. Guerra, and J. de Lara, "Quick fixing ATL transformations with speculative analysis," Software and Systems Modeling, vol. 17, no. 3, pp. 779-813, 2018.

[12] — - "AnATLyzer: an advanced IDE for ATL model transformations," in ICSE'18 Companion Proceedings. ACM, 2018, pp. $85-88$

[13] F. Jouault, "Regression Tests for the ATL Virtual Machine," 2013. [Online]. Available: https://wiki.eclipse.org/ATL_VM_ Testing

[14] C. Eastman, P. Tiecholz, R. Sacks, and K. Liston, BIM Handbook: A Guide to Building Information Modeling for Owners, Managers, Designers, Engineers and Contractors, 2nd ed. John Wiley, 2011.
[15] Y. Dajsuren and M. van den Brand, Eds., Automotive Systems and Software Engineering - State of the Art and Future Trends. Springer, 2019.

[16] J. Troya, H. Brunelière, M. Fleck, M. Wimmer, L. OrueEchevarria, and J. Gorroñogoitia, "ARTIST: Model-Based Stairway to the Cloud," in Proc. of the Projects Showcase @ STAF, vol. 1400. CEUR-WS.org, 2015, pp. 1-8.

[17] Object Management Group, Object Constraint Language (OCL) Specification. Version 2.2, Feb. 2010, OMG Document formal/2010-02-01.

[18] D. Wagelaar, M. Tisi, J. Cabot, and F. Jouault, "Towards a general composition semantics for rule-based model transformation," in Proc. of MODELS 2011, ser. LNCS, vol. 6981. Springer, 2011, pp. 623-637.

[19] D. Kolovos, L. Rose, R. Paige, and A. García-Domínguez, The Epsilon Book. Eclipse, 2010. [Online]. Available: https: //www.eclipse.org/epsilon/doc/book/

[20] L. Burgueño, M. Wimmer, and A. Vallecillo, "A Linda-based platform for the parallel execution of out-place model transformations," Information and Software Technology, vol. 79, pp. 1735, 2016.

[21] J. S. Cuadrado, F. Jouault, J. G. Molina, and J. Bézivin, "Optimization patterns for OCL-based model transformations," in Proc. of Workshops and Symposia at MODELS 2008, ser. LNCS, vol. 5421. Springer, 2008, pp. 273-284.

[22] A. S. Lee, "A scientific methodology for MIS case studies," MIS Quarterly, vol. 13, no. 1, pp. 33-50, 1989.

[23] P. Runeson and M. Höst, "Guidelines for Conducting and Reporting Case Study Research in Software Engineering," Empirical Software Engineering, vol. 14, no. 2, pp. 131-164, 2009.

[24] "AnATLyzer tutorial," 2017, https://github.com/jesusc/ anatlyzer-models17

[25] A. Kusel, J. Schönböck, M. Wimmer, G. Kappel, W. Retschitzegger, and W. Schwinger, "Reuse in model-to-model transformation languages: are we there yet?" Software and Systems Modeling, vol. 14, no. 2, pp. 537-572, 2015.

[26] T. Hoefler and R. Belli, "Scientific benchmarking of parallel computing systems: twelve ways to tell the masses when reporting performance results," in Proc. of SC'15. ACM, 2015, pp. 73:1$73: 12$.

[27] R. Wei, D. S. Kolovos, A. Garcia-Dominguez, K. Barmpis, and R. F. Paige, "Partial loading of XMI models," in Proc. of MODELS 2016. ACM, 2016, pp. 329-339.

[28] C. Wohlin, P. Runeson, M. Höst, M. C. Ohlsson, and B. Regnell, Experimentation in Software Engineering. Springer, 2012.

[29] G. Szárnyas, B. Izsó, I. Ráth, and D. Varró, "The train benchmark: cross-technology performance evaluation of continuous model queries," Software \& Systems Modeling, vol. 17, no. 4, pp. 1365-1393, 2018.

[30] N. Kahani, M. Bagherzadeh, J. R. Cordy, J. Dingel, and D. Varró, "Survey and classification of model transformation tools," Software and Systems Modeling, vol. 18, no. 4, pp. 23612397, 2019.

[31] T. L. Calvar, F. Jouault, F. Chhel, and M. Clavreul, "Efficient ATL incremental transformations," Journal of Object Technology, vol. 18, no. 3, pp. 2:1-17, 2019.

[32] A. Razavi and K. Kontogiannis, "Partial evaluation of model transformations," in Proc. of ICSE 2012. IEEE Computer Society, 2012, pp. 562-572.

[33] D. Varró, G. Bergmann, Á. Hegedüs, Á. Horváth, I. Ráth, and Z. Ujhelyi, "Road to a reactive and incremental model transformation platform: three generations of the VIATRA framework," Software and Systems Modeling, vol. 15, no. 3, pp. 609-629, 2016.

[34] M. Tisi, S. M. Perez, F. Jouault, and J. Cabot, "Lazy execution of model-to-model transformations," in Proc. of MODELS 2011, ser. LNCS, vol. 6981. Springer, 2011, pp. 32-46.

[35] J. S. Cuadrado and J. de Lara, "Streaming model transformations: Scenarios, challenges and initial solutions," in Proc. of ICMT 2013, ser. LNCS, vol. 7909. Springer, 2013, pp. 1-16.

[36] A. Bergmayr, J. Troya, and M. Wimmer, "From out-place transformation evolution to in-place model patching," in Proc. of ASE 2014. ACM, 2014, pp. 647-652.

[37] L. C. Camargo and M. D. D. Fabro, "Applying a data-centric framework for developing model transformations," in Proc. of SAC 2019. ACM, 2019, pp. 1570-1573. 
[38] L. Burgueño, "Concurrent and Distributed Model Transformations based on Linda," in Proc. of the MODELS 2013 Doctoral Symposium, vol. 1071. CEUR-WS.org, 2013, pp. 9-16.

[39] G. Szárnyas, B. Izsó, I. Ráth, D. Harmath, G. Bergmann, and D. Varró, "IncQuery-D: A Distributed Incremental Model Query Framework in the Cloud," in Proc. of MODELS 2014, ser. LNCS, vol. 8767. Springer, 2014, pp. 653-669.

[40] G. Bergmann, "Incremental model queries in model-driven design," Ph.D. dissertation, Budapest University of Technology and Economics, 2013.

[41] M. van Amstel, S. Bosems, I. Kurtev, and L. F. Pires, "Performance in model transformations: Experiments with ATL and QVT," in Proc. of ICMT 2011, ser. LNCS, vol. 6707. Springer, 2011, pp. 198-212.

[42] M. Wimmer, S. M. Perez, F. Jouault, and J. Cabot, "A catalogue of refactorings for model-to-model transformations," Journal of Object Technology, vol. 11, no. 2, pp. 2: 1-40, 2012.

[43] G. Daniel, F. Jouault, G. Sunyé, and J. Cabot, "Gremlin-ATL: a scalable model transformation framework," in Proc. of $A S E$ 2017. IEEE Computer Society, 2017, pp. 462-472.

[44] M. Besta, E. Peter, R. Gerstenberger, M. Fischer, M. Podstawski, C. Barthels, G. Alonso, and T. Hoefler, "Demystifying graph databases: Analysis and taxonomy of data organization, system designs, and graph queries," CoRR, vol. abs/1910.09017, 2019. [Online]. Available: http://arxiv.org/abs/1910.09017

[45] T. Kersten, V. Leis, A. Kemper, T. Neumann, A. Pavlo, and P. A. Boncz, "Everything you always wanted to know about compiled and vectorized queries but were afraid to ask," Proc. VLDB Endow., vol. 11, no. 13, pp. 2209-2222, 2018.

[46] G. Bergmann, I. Ráth, and D. Varró, "Parallelization of graph transformation based on incremental pattern matching," Electronic Communication of the European Association of Software Science and Technology (ECEASST), vol. 18, 2009.

[47] G. Imre and G. Mezei, "Parallel graph transformations on multicore systems," in Proc. of MSEPT 2012, ser. LNCS, vol. 7303. Springer, 2012, pp. 86-89.

[48] C. Krause, M. Tichy, and H. Giese, "Implementing graph transformations in the bulk synchronous parallel model," in Proc. of FASE 2014, ser. LNCS, vol. 8411. Springer, 2014, pp. 325-339.

[49] D. S. Kolovos, R. F. Paige, and F. A. C. Polack, "The Epsilon Transformation Language," in Proc. of ICMT 2008, ser. LNCS, vol. 5063. Springer, 2008, pp. 46-60.

[50] S. Madani, D. S. Kolovos, and R. F. Paige, "Towards optimisation of model queries: A parallel execution approach," Journal of Object Technology, vol. 18, no. 2, pp. 3:1-21, 2019.

[51] - "Parallel Model Validation with Epsilon," in Proc. of ECMFA 2018, ser. LNCS, vol. 10890. Springer, 2018, pp. 115131.

[52] A. Bucchiarone, J. Cabot, R. F. Paige, and A. Pierantonio, "Grand challenges in model-driven engineering: an analysis of the state of the research," Software and Systems Modeling, vol. 19 , no. 1 , pp. $5-13,2020$.

[53] E. Bousse and M. Wimmer, "Domain-Level Observation and Control for Compiled Executable DSLs," in Proc. of MODELS 2019. IEEE, 2019, pp. 150-160.

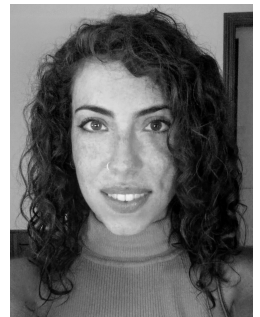

Loli Burgueño is a postdoctoral researcher and lecturer at the Open University of Catalonia (Spain) and CEA LIST (France). Her research interests focus on Model-Driven Engineering, in particular the performance, scalability and testing of model transformations, the modeling of uncertainty in software models for its use in the Industry 4.0 and the integration of Artificial Intelligence techniques into modeling tools and processes. For more information, please visit https://som-research.uoc.edu/loli-burgueno/

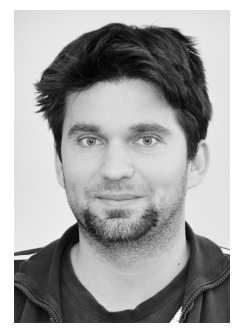

Manuel Wimmer is full professor leading the Institute of Business Informatics-Software Engineering at the Johannes Kepler University Linz, and he is head of the Christian Doppler Laboratory CDL-MINT. His research interests comprise foundations of model engineering techniques as well as their application in domains such as tool interoperability, legacy tool modernization, model versioning and evolution, and industrial engineering. For more information, please visit https://www.se.jku.at/manuel-wimmer

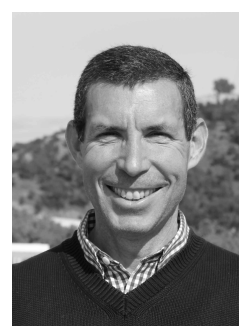

Antonio Vallecillo is Full Professor of Software Engineering at the University of Málaga, Spain, where he leads the Atenea research group dedicated to systems modeling and analysis. His research interests include open distributed processing, model-based software engineering, and software quality. For more information, please visit http://www.lcc.uma.es/ av

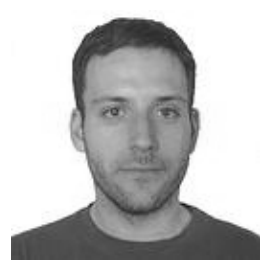

Jesús Sánchez Cuadrado is a Ramón y Cajal researcher at Universidad de Murcia. Previously he was an associate professor at Universidad Autónoma de Madrid. His research is focused on Model Driven Engineering (MDE) topics, notably model transformation languages, metamodelling and domain specific languages. On these topics, he has published several articles in journals and peer-reviewed conferences, and developed several tools. For more information, please visit http://sanchezcuadrado.es. 